\title{
Item Collection
}

National Cancer Institute

\section{Source}

National Cancer Institute. Item Collection. NCI Thesaurus. Code C45261.

A group of items of the same type, gathered for display or study. 